\title{
Temporoparietal and Infratemporal fossa abscess as a complication of dental extraction - a rare and potentially lethal condition
}

\author{
Probal Chatterji', Isha Goyal ${ }^{2}$ \\ ${ }^{1}$ Professor \& Head, ${ }^{2}$ Senior Resident, Department of Otorhinolaryngology \& Head-Neck Surgery, Teerthanker \\ Mahaveer Medical College \& Research Center, Moradabad, Uttar Pradesh - 244001, India
}

Abscesses of the infratemporal fossa are rare in clinical practice. Usually it occurs as a complication of odontogenic infections, dental extraction, sinusitis and trauma. The initial signs \& symptoms of this condition may be very non-specific in nature. If treatment is delayed, the abscess may extend superiorly to involve the temporoparietal region and only then it can be appreciated clinically. A middle aged male patient presented to us with the complaint of restricted mouth opening along with extensive swelling of the temporoparietal region. There was past history of tooth extraction. MRI scan confirmed it to be a case of temporoparietal \& infratemporal fossa abscess with features of osteomyelitis of the ramus and adjacent body of the mandible on the right side. The patient was started on parenteral antibiotics and subsequently the abscess was drained by combined external and intraoral approach. The patient had an uneventful recovery. A thorough review of the literature has been done in relation to this condition and its management. A delay in diagnosis of this condition can lead to a number of potentially lethal complications. CT scan and MRI scan are the best modalities for confirming the diagnosis in suspected cases. Prompt drainage of the abscesses under antibiotic cover is the cornerstone of management. Isolated abscess of the infratemporal fossa may be drained intraorally or endoscopically by trans-maxillary approach. However if the temporoparietal region is also involved, a combined approach with a separate incision over the temporal region is indicated.

Key words: Infratemporal abscess; Odontogenic infection; Osteomyelitis jaw; Temporoparietal abscess

\section{INTRODUCTION}

The infratemporal fossa is a relatively closed irregularly shaped space bounded above by the infratemporal surface of the greater wing of sphenoid and part of the temporal bone, medially by the lateral plate of the pterygoid process of the sphenoid and anteriorly by the posterior surface of the maxilla. The inferior portion of the infratemporal fossa is called the pterygomandibular space which is bounded medially by the lower part of the medial pterygoid muscle and laterally by the ramus of the mandible. Superiorly

Address for correspondence:

Dr Probal Chatterji, Professor \& Head - Department of Otorhinolaryngology, Teerthanker Mahaveer Medical College \& Research Center Bagadpur, Delhi Road, Moradabad - 244001, Uttar Pradesh, India Mobile: +91 7523829592

E-mail: probalch@hotmail.com 
of tooth extraction, maxillary sinusitis, traumatic fracture of the maxillary sinus wall and temporomandibular arthroscopy. ${ }^{2}$ A search of the available literature reveals that although it is a recognized clinical entity, only a handful of cases of abscess involving the temporoparietal and infratemporal fossa have been reported till date. ${ }^{3,4}$ Signs $\&$ symptoms of this condition may be very nonspecific in nature leading to confusion in diagnosis and it can progress to certain life threatening complications if left untreated. ${ }^{5}$

We present a case report of a patient with temporoparietal and infratemporal fossa abscess following molar extraction of the lower jaw.

\section{CASE DESCRIPTION}

A 50 year old male patient presented with the chief complaint of restricted mouth opening for four weeks duration. This was followed by an associated complaint of gradual swelling over the right side of the head for the last three weeks along with low grade fever. The patient gave a history of extraction of the third molar of the lower right jaw just a few days prior to the onset of symptoms. On examination, there was a diffuse swelling (approximately $7.5 \times 7.5 \mathrm{~cm}$ ) involving the temporoparietal region of the head (Figure 1). The temperature of the skin over the swelling was slightly raised compared to the other areas. The swelling was soft, non-compressible, non-pulsatile and slightly tender on palpation. The mouth opening was restricted and the gum over the posterior aspect of the right lower jaw was swollen and discharging small amounts of pus. Overall dental hygiene was poor. There was slight tenderness over the right masseteric region on palpation. No cervical lymphadenopathy was detected. The rest of the clinical examination was unremarkable. Past medical records showed that he had been successfully treated for pulmonary tuberculosis a year ago. A clinical diagnosis of masticator space infection along with abscess of the temporoparietal space was provisionally made. MRI scan of the head with contrast was advised and it showed features suggestive of osteomyelitis of the ramus and adjacent body of the mandible on the right side along with edema and heterogeneous post contrast enhancement of the muscles of mastication of the right side. An associated large, complex multiloculated collection was noted in the right infratemporal fossa along with superior extension within the extra-calvarial scalp tissues along the fascial plane in the right temporoparietal region suggestive of an abscess formation (Figures $2 \& 3$ ). Thus a definitive diagnosis of abscess of the right temporoparietal and infratemporal fossa as a complication of dental extraction was finally arrived at.

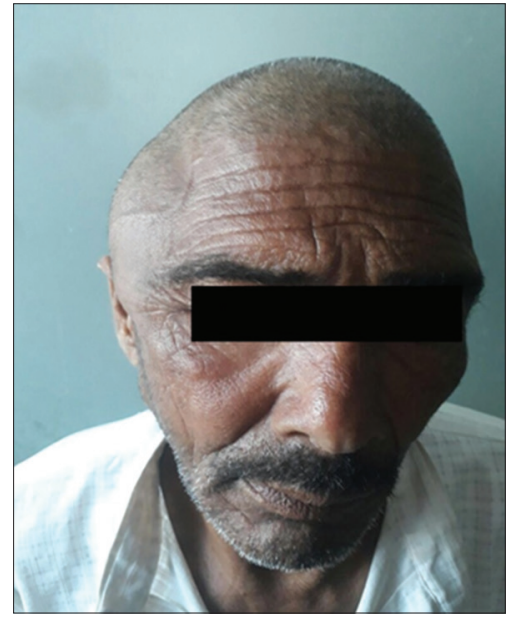

Figure 1: Photograph of the patient showing diffuse swelling of the right temporoparietal region

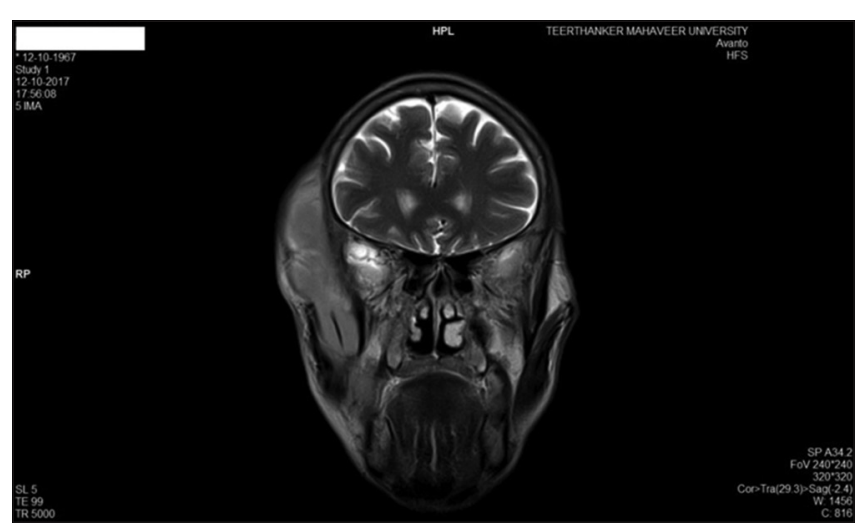

Figure 2: MRI scan (coronal cut) showing involvement of the right temporoparietal and infratemporal fossa

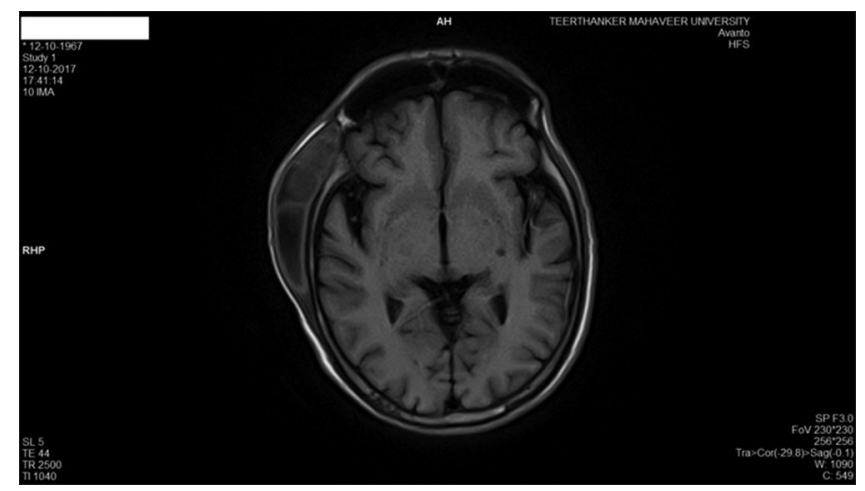

Figure 3: MRI scan (axial cut) showing the multiloculated abscess involving the temporoparietal region

The patient was admitted and administered a combination of amoxicillin and clavulanic acid along with metronidazole by intravenous route. He was also counseled regarding the necessity of incision and drainage of the abscess and prepared accordingly. On investigation, the blood hemoglobin level was $10.3 \mathrm{gm} \%$ and total leukocyte count 
was raised (17000/cu mm of blood) along with neutrophilia $(79 \%)$. All other routine tests were within normal limits.

The abscess was drained under general anesthesia by a combination of intraoral approach and temporal approach for draining the infratemporal space and the temporoparietal space respectively. The intraoral incision was given laterally in the gingivolabial groove over the anterior aspect of the ascending ramus of the mandible and a hemostat was introduced to drain the collection in the infratemporal fossa. The other incision was applied vertically about $2.5 \mathrm{~cm}$ in length on the skin of the temporal region just above the zygomatic arch just behind the orbital process of the zygomatic bone. Again a hemostat was introduced to break the loculi and drain the abscess. Finally a small drain was inserted and a dressing with tight roller bandage was applied over the scalp. The dressing was changed daily and the drain was removed after 72 hours. An oral antiseptic rinse (Betadine mouth wash) was also advised for maintenance of oral hygiene. Patient was shifted to oral medication after 5 days and discharged with advice to perform mouth opening exercises. He had a slow and steady recovery with adequate mouth opening after about 4 weeks.

\section{DISCUSSION}

Isolated abscess of the infratemporal fossa following dental extraction has been rarely reported in the literature. ${ }^{3}$ Even though both the jaws have been implicated, in most cases the tooth responsible was a mandibular molar. ${ }^{6}$ However, combined abscess of the temporoparietal and infratemporal fossa complicating dental extraction are rarer still with even fewer references. ${ }^{1,4}$

Since the infratemporal space is a relatively closed space, an abscess of this region rarely causes a visible swelling. Hence an isolated infection of the infratemporal space is a difficult condition to diagnose., ${ }^{5,6}$ The abscess can be appreciated visually and palpated clinically only when it extends upwards to involve the temporoparietal region. Early cases may thus be missed easily or misdiagnosed leading to potentially lethal complications due to spread of infection along different routes. ${ }^{7}$ The possible complications of this condition include necrotizing fasciitis, respiratory obstruction, descending mediastinitis, pericarditis, brain abscess, orbital involvement and sepsis., ${ }^{2,5}$ Therefore, the clinician must always consider this condition in the differential diagnosis whenever a patient presents with excessive local pain and trismus following recent history of odontogenic infection, dental extraction, maxillary trauma etc. Associated features of raised total leukocyte count, neutrophilia and raised CRP levels even in the absence of obvious local swelling should raise further suspicion. The common conditions from which this entity must be differentiated include parotitis \& temporomandibular joint disorders. ${ }^{5}$ It is recommended that Computed Tomography (CT) or Magnetic Resonance Imaging (MRI) should be performed in suspected cases as they are the best modalities to clinch the diagnosis. The clinician should not wait for signs like swelling of the temporal region to develop due to the risk of complications as discussed above. ${ }^{6}$ Once the condition is diagnosed, treatment must be instituted at the earliest in order to prevent the progress to the above named complications. ${ }^{7}$

Treatment includes the administration of appropriate intravenous antibiotics and drainage of the abscess. Culture of the pus has not shown any advantage in terms of antibiotic selection as most culture reports have shown polymicrobial growths. ${ }^{5}$ Any other medical condition like uncontrolled diabetes which can complicate the picture must also be managed simultaneously. Surgical drainage of the pus remains the cornerstone of management. However, as the infratemporal fossa is a relatively inaccessible space, it is difficult to secure a route for drainage of the accumulated pus. ${ }^{8}$ Theoretically, the space may be approached via various routes like advancing from inside the mouth, from the maxillary sinus, from the medial side of the parotid gland etc. The disadvantage with all these approaches lies in the fact that a clear field of view is all but impossible during incision and drainage. ${ }^{6}$ Among all these approaches, the intraoral approach has been used widely over the years for access when the abscess is restricted to the infratemporal fossa alone. ${ }^{9}$ But recently in 2014, endoscopic drainage of the infratemporal fossa abscess via the trans-maxillary approach has been reported for the first time in the literature..$^{10}$ Most likely this approach will become the most preferred one in the years to come because of its minimally invasive technique and excellent visualization. However if the temporoparietal space is also concomitantly involved, then a separate approach must be made to drain the pus accumulated in the upper compartment. To achieve that purpose, an incision over the temporal region as described above is probably the most effective. Thus if the abscess involves both the compartments, a combined approach to drain the pus will work best as per our opinion.

\section{CONCLUSION}

Temporoparietal and Infratemporal fossa abscesses are uncommon in clinical practice. The rarity of this condition, non-specific signs \& symptoms as well as the potential for progress towards lethal complications makes the diagnosis of this condition a challenge for the clinician. CT scan and MRI scan are the best modalities for confirming the 
diagnosis in suspected cases. An early diagnosis followed by prompt medical and surgical management is of paramount importance for a successful outcome. Isolated abscess of the infratemporal fossa may be drained by intraoral approach or endoscopically by the trans-maxillary approach. However if the temporoparietal region is also involved, a combined approach with a separate incision over the temporal region is indicated to drain the collection.

\section{REFERENCES}

1. Diacono MS and Wass AR. Infratemporal and temporal fossa abscess complicating dental extraction. J Accid Emerg Med 1998; 15(1): 59-61.

2. Leventhal $D$ and Schwartz DN. Infratemporal Fossa Abscess - Complication of Dental Injection. Arch Otolaryngol Head Neck Surg 2008; 134(5): 551-553.

3. Kamath MP, Bhojwani KM, Mahale A, Meyyappan $H$ and Abhijit K. Infratemporal fossa abscess: A diagnostic dilemma. Ear Nose Throat J 2009; 88(5): E23.

4. Pillai AK, Kulkarni P, Moghe S, Vishnu V, Yadav SD and
Dastagir SS. Infra-temporal \& temporal abscess - Retrograde infection from mandibular molars. IOSR Journal of Dental and Medical Sciences 2014; 13(11): 96-99.

5. Mesgarzadeh AH, Ghavimi MA, Gok $G$ and Zarghami A. Infratemporal space infection following maxillary third molar extraction in an uncontrolled diabetic. Journal of Dental Research, Dental Clinics, Dental Prospects 2012; 6(3): 113-115.

6. Kasahara K, Ogawa C, Matsuzaka K, Yamamura T, Takano M, Saitou C, et al. A case of infratemporal fossa abscess with signs of chronic maxillary osteomyelitis. Bull Tokyo Dent Coll. 2015; 56(2): 121-129.

7. Emes $Y$, Yalcin S, Aybar B and Bilici IS. Infratemporal fossa infection with inferior alveolar nerve involvement. J Istanb Univ Fac Dent 2016; 50(3): 46-50.

8. Kawada S, Uekubo I, Asano T and Takahashi N. Two cases of Infratemporal Fossa Abscess. Tokeibu Geka 2008; 18: 119-125. (in Japanese)

9. Schwimmer AM, Roth SE and Morrison SN. The use of computerized tomography in the diagnosis and management of temporal and infratemporal space abscesses. Oral Surg Oral Med Oral Pathol 1988; 66: 17-20.

10. Sundaram SS, Rajan P and Balasubramanian A. Endoscopic transmaxillary drainage of an infratemporal fossa abscess. BMJ Case Rep 2014; pii: bcr2013200637.

\footnotetext{
Author's Contribution:

PC- Concept and design of the study, reviewed the literature, manuscript preparation and critical revision of the manuscript; IG- Concept, collected data and review of literature and helped in preparing first draft of manuscript.

Orcid ID:

Dr Probal Chatterji - (D) http://orcid.org/0000-0003-4839-0696, Dr Isha Goyal - (i) https://orcid.org/0000-0002-0563-8551

Work attributed to: Department of Otorhinolaryngology \& Head-Neck Surgery, Teerthanker Mahaveer Medical College \& Research Center, Moradabad, Uttar Pradesh - 244001, India
}

Source of Support: Nil, Conflict of Interest: None declared. 